\title{
Effects of laser treatment on respiratory function in malignant narrowing of the central airways
}

\author{
JJ GILMARTIN, D VEALE, BG COOPER, PM KEAVEY, GJ GIBSON, \\ GN MORRITT
}

From the Departments of Respiratory Medicine, Cardiothoracic Surgery, and Medical Physics, Freeman Hospital, Newcastle upon Tyne

ABSTRACT Twenty patients with inoperable carcinoma in the trachea or a main bronchus wereo investigated before and one and 10 days after treatment with a carbon dioxide laser. Patients were assessed by spirometry, maximum flow-volume loops, and a visual analogue score of breathlessness on a scale from 0 (not at all breathless) to 100 (very breathless). At day 10 mean $\mathrm{FEV}_{1}$ had improved from $51.9 \%$ to $62.6 \%$ of predicted $(\mathrm{p}<0.02)$ and mean peak expiratory flow (PEF) from $45 \cdot 3 \%$ to $53.1 \%$ of the predicted value $(\mathrm{p}<0.05)$. Improvements in maximum inspiratory and expiratory flows at $50 \%$ vital capacity were not significant but the breathlessness score decreased from a meano of 49.1 to 35.3 ( $p<0.01$ ). Improvements in breathlessness were significantly correlated with increases in $\mathrm{FEV}_{1}$ and PEF. Thirteen of the 20 patients had unilateral tumours with partial oro complete occlusion of the main bronchus; in these perfusion and ventilation were assessed bys radioisotope scans before and 10 days after treatment. Seven of the 13 patients showed an increase in perfusion of the affected lung after treatment but the improvement was small, with a meano increase in unilateral perfusion in the 13 patients of $2.4 \%$ of the total counts. Four patients with no perfusion of the affected side showed no significant improvement after laser treatment. Changes ing ventilation scans were similar to those in perfusion. It is concluded that laser treatment improvesairway function and dyspnoea in malignant narrowing of central airways and that in unilaterap̦ obstruction such treatment results, at best, in a small increase in the contribution of the affected lung to perfusion.

Phototherapy by laser has recently been applied in the treatment of bronchial carcinoma via the bronchoscope. ${ }^{12}$ Previous reports have concentrated on technique or on the effect on survival ${ }^{12}$ and little information has been published on changes in respiratory function after laser treatment.

The likely benefit from removal of tracheal lesions by laser is self evident, ${ }^{1}$ but its use in unilateral lesions, where one or other main bronchus is occluded, has the potential disadvantage of increasing the ventilation of underperfused lung. It is well recognised that defects of ventilation and perfusion on isotope scans are often surprisingly large in patients with centrally placed carcinomas. ${ }^{34}$ We have studied the effects of laser treatment of malignant narrowing of the trachea or of a main bronchus on ventilatory

Address for correspondence: Dr J J Gilmartin, Royal Infirmary, Sunderland SR2 7JE. (Reprints will not be available.)

Accepted 23 February 1987 function and breathlessness. In addition, in patientş่ with tumour confined to one or other main bronchus we performed scans before and after treatment to assess how much improvement in ipsilateral function? results from phototherapy.

\section{Patients and methods}

Twenty patients with malignant disease proved an bronchoscopy were referred for laser treatment because of distressing breathlessness (table 1). Elever of the patients had had prior radiotherapy but in 19 this had been given more than one year before lase treatment; the other patient had had radiotherapy one month before the study.

The severity of airflow obstruction was assessed b $\vec{D}$ measurements of $F E_{1}$ and by maximum inspirator and expiratory flow-volume curves. The peaf expiratory flow (PEF) and the maximum flows at mix vital capacity ( $\dot{\mathrm{V}}_{\mathrm{Emax}_{\mathbf{5}}}$ and $\dot{\mathrm{V}}_{\operatorname{Imax}}$ ) were taken as the 
Effects of laser treatment on respiratory function in malignant narrowing of the central airways

Table 1 Patients' details and pulmonary function values (\% predicted) before laser treatment

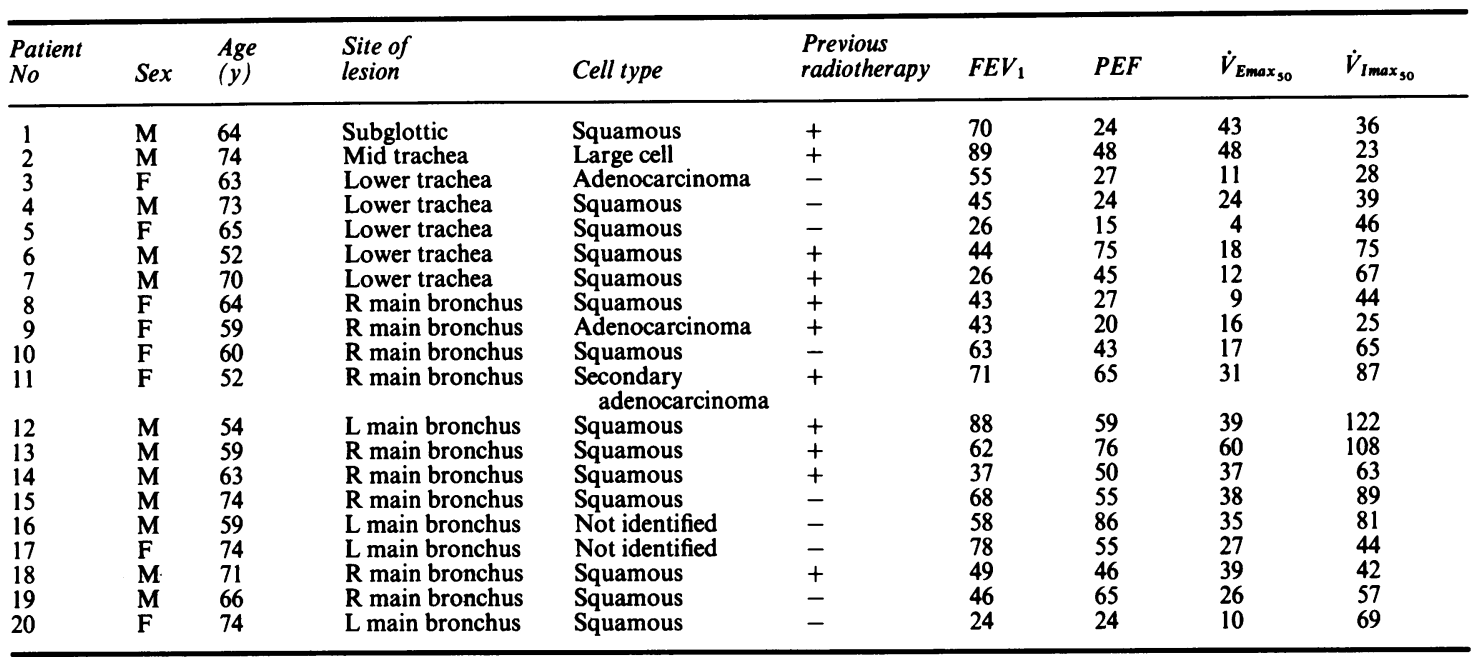

$\mathrm{R}$-right; $\mathrm{L}$-left; PEF-peak expiratory flow; $\dot{\mathrm{VE}}_{\mathbf{s o}}, \dot{\mathrm{V}}_{\mathrm{so}}$-maximum expiratory and inspiratory flow at mid vital capacity.

highest values obtained from an "envelope" of three technically satisfactory flow-volume loops. ${ }^{5}$ Each measurement was expressed as a percentage of predicted normal on the basis of standard regression equations. ${ }^{56}$ Breathlessness was assessed by using a $100 \mathrm{~mm}$ visual analogue scale marked "not at all breathless" (0) at one end and "very breathless" (100) at the other.

Laser resection was performed under general anaesthesia by one operator (GNM) with a carbon dioxide laser and forceps through a modified rigid bronchoscope. As much tumour as could safely be removed was resected in a single session. Seven patients were taking prednisolone at the time of laser treatment and all patients were given dexamethasone during induction of anaesthesia.

Functional assessment was performed before, the day after, and 10 days after laser treatment.

Thirteen of the 20 patients had unilateral disease and in these patients relative perfusion and ventilation of each lung was assessed by radioisotope scanning, with ${ }^{99 m} \mathrm{Tc}$ and ${ }^{133} \mathrm{Xe}$ respectively, before and 10 days after treatment. The seven patients (Nos 1-7) with disease limited to the trachea did not have lung scans.

The contribution of the affected lung to total perfusion was calculated by computer from the average counts obtained in both posteroanterior and anteroposterior views and expressed as

$$
\frac{\text { perfusion counts from affected lung }}{\text { perfusion counts from both lungs }} \times 100 \text {. }
$$

In patients 8-12 the contribution of the diseased lung to total ventilation was assessed qualitatively from the initial full inhalation scan. In patients 13-20 the contribution to ventilation from the affected side was assessed quantitatively from the inhalation scan and expressed as

$$
\frac{\text { ventilation counts from affected lung }}{\text { ventilation counts from both lungs }} \times 100 \text {. }
$$

Differences before and after treatment were examined by means of paired $t$-tests. Differences between patients with lesions in the trachea (Nos 1-7) and those with tumour in either main bronchus were examined by means of unpaired $t$-tests.

\section{Results}

All the patients had some degree of airflow limitation before treatment, with a mean $\mathrm{FEV}_{1}$ of $59 \cdot 1 \%$ of the predicted value (table 1 ). The changes in the chest radiograph and lung scan (in patients 8-20) after treatment are shown in table 2 . The changes in functional data at day 1 are shown in figure 1 and at day 10 in figure 2.

\section{DAY 1}

Changes in function were variable and only the change in $\mathrm{FEV}_{1}$ was significant ( $p<0.05$; fig 1). Only eight patients recorded a symptomatic improvement on day 1 by the visual analogue scale scores and the remaining 12 showed a deterioration.

DAY 10

Two of the patients did not complete the investigations on day 10 (fig 2), one (No 4) because of an intercurrent infection. The other (No 8) did not attend for functional assessment but did have lung scans 
Table 2 Effect of laser treatment on chest radiographs and unilateral lung function

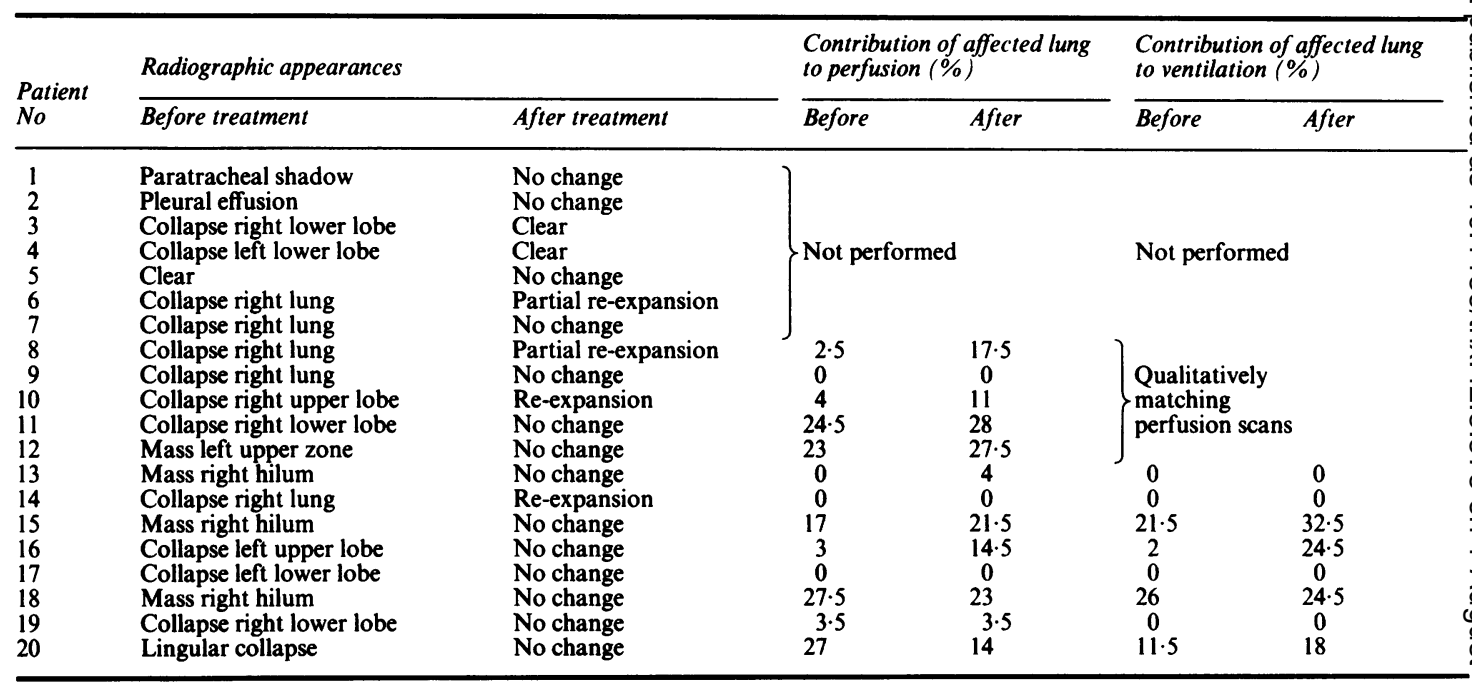

performed. All the other patients showed some improvement in at least one of the indices of airway function. Significant changes were seen in both $\mathrm{FEV}_{1}$ $(p<0.02)$ and PEF $(p<0.05)$; but the absolute changes were small $\left(\triangle F E V_{1}\right.$ : mean $\left.(S D) 0.36(0.37) 1\right)$, range -0.3 to $+1.01 ; \Delta$ PEF $39(66.4) 1 \mathrm{~min}^{-1}$, range
-85 to $+2101 \min ^{-1}$ ). The changes in $\dot{\mathrm{V}}_{\text {Emax so }}$ and. $\dot{V}_{\text {Imax }}$ were more variable and not significant. The change in PEF, $\mathrm{FEV}_{1}$, and $\dot{\mathrm{V}}_{\mathrm{Emax}_{\text {so }}}$ were all significantly greater $(p<0.05)$ in those patients witho tracheal lesions than in those with main bronchuso lesions.

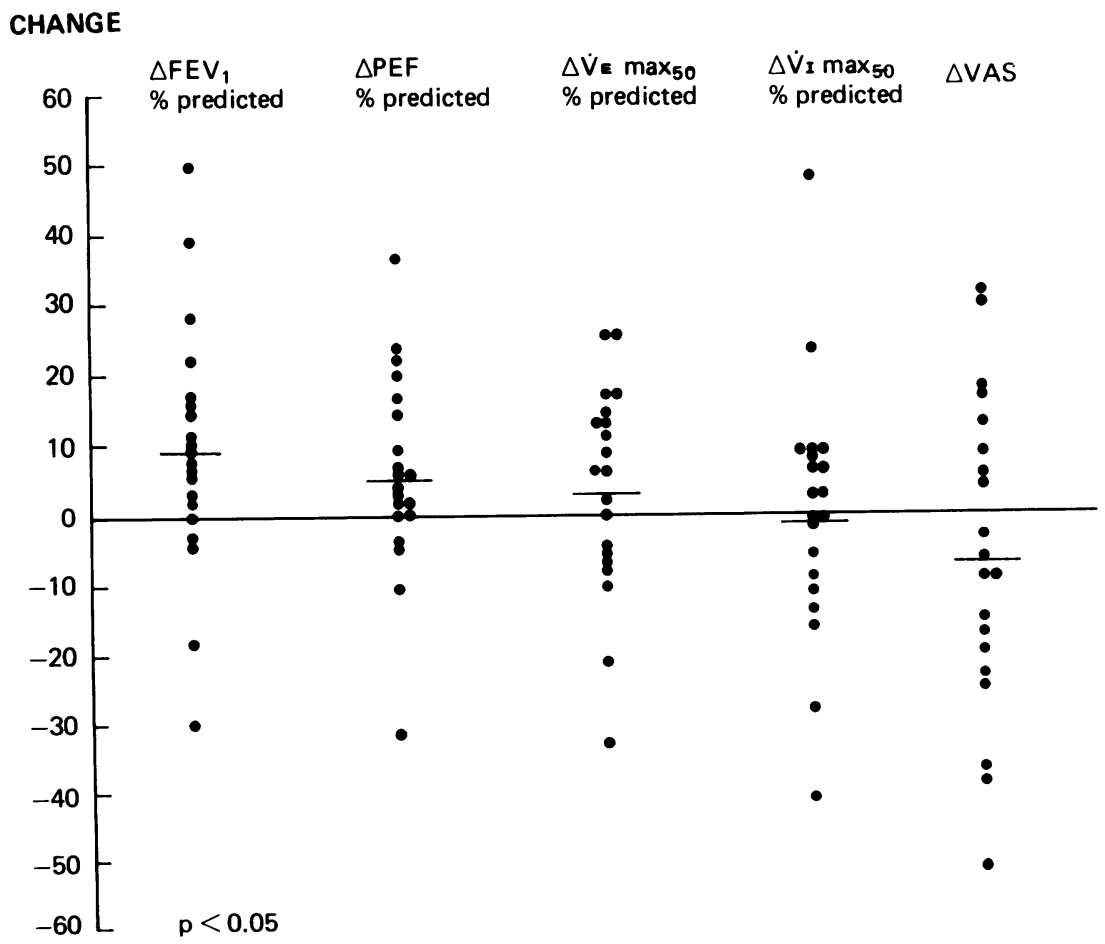

Fig 1 Changes in lung function and breathlessness score in all patients on day 1 after laser treatment. The change in each functional index-FEV $V_{1}$, peak expiratoryo flow (PEF), maximum flow at mid vital capacity

( $\dot{V}_{\max _{30}}$ )-represents the difference between the pretreatment value and the measurement on day 1 (each expressed as \% predicted value). The change in breathlessness score is the difference between visual analogue scale (VAS) scores ( $\mathrm{mm}$ ) before and after treatment. Bars represent meat values. Only the change in $F E V$, was statistically significant $(p<0.05)$. 
Effects of laser treatment on respiratory function in malignant narrowing of the central airways

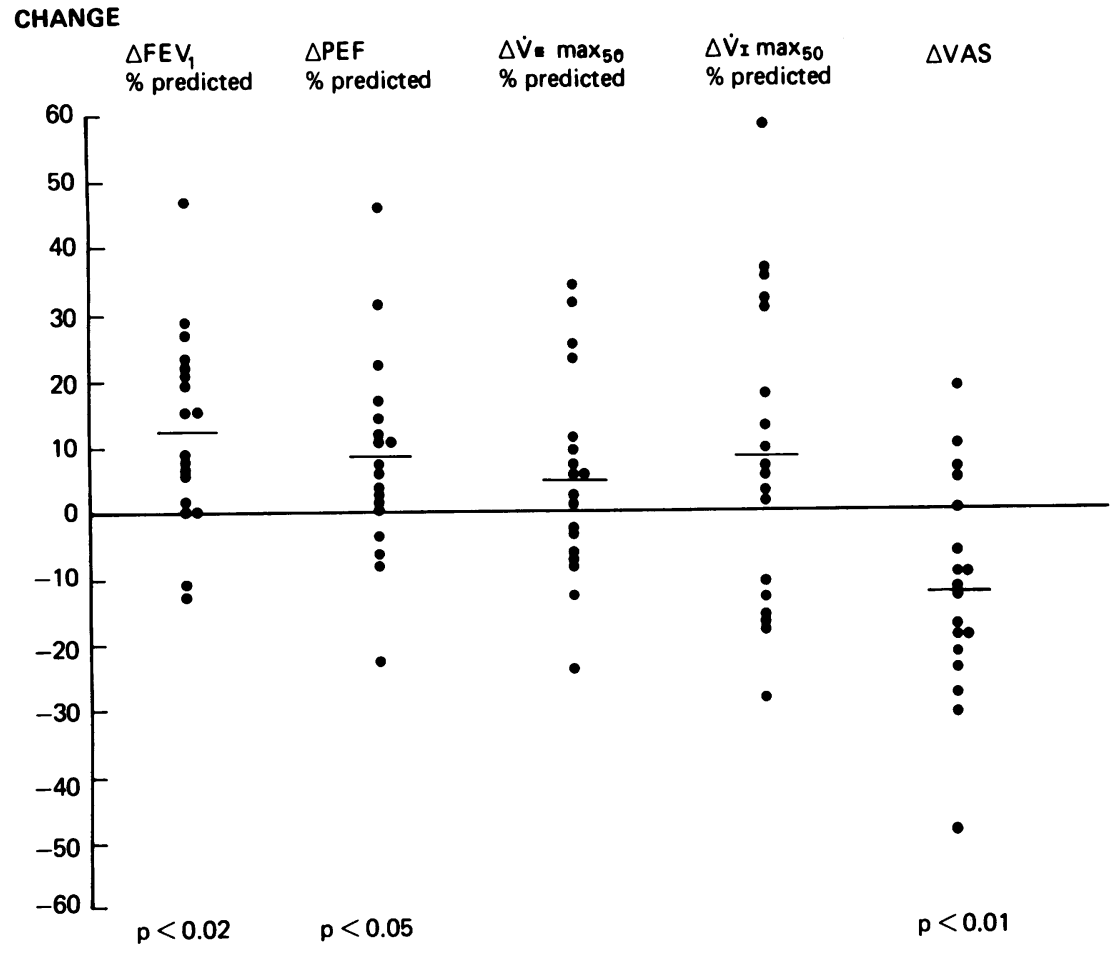

Fig 2 Changes in lung function and breathlessness score in 18 patients on day 10 after laser treatment.

Conventions as in figure 1.

There were significant improvements in $F E V_{1}, P E F$, and breathlessness score.

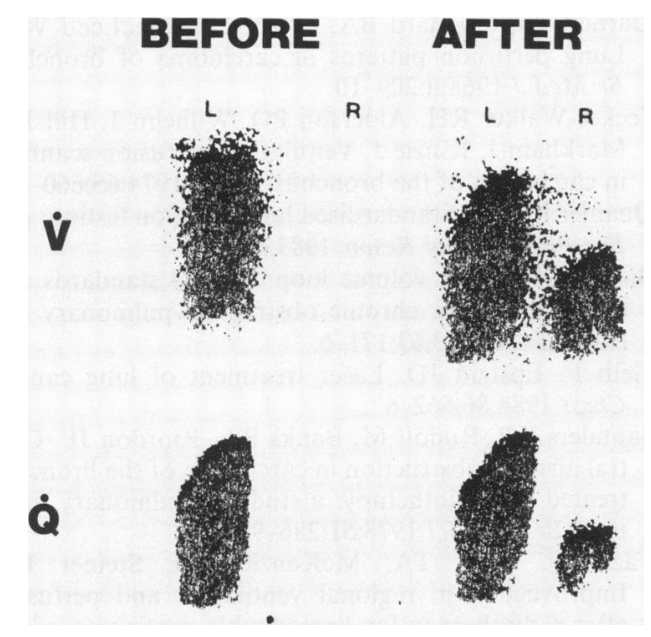

Fig 3 Ventilation ( $\dot{V}$, upper) and perfusion ( $\dot{Q}$, lower) posterior anterior scans before (left) and after (right) laser treatment in patient 8 . Ventilation scans represent counts obtained after a single full inspiration of gas containing xenon-133. Before treatment there was very little $\dot{V}$ or $\dot{Q}$ of the right lung; after treatment the contribution of the right lung to perfusion was $17.5 \%$ of the total and the distribution of ventilation was similar.

Four patients indicated increased breathlessness at this stage. In the group as a whole the changes in visual analogue scale scores correlated with the changes in $\mathrm{FEV}_{1}(\mathrm{r}=-0.52 ; \mathrm{p}<0.05)$ and PEF $(\mathrm{r}=-0.63: \mathrm{p}<0.01)$.

In six patients (Nos $3,4,6,8,10,14$ ) the chest radiograph showed partial or complete re-expansion of a collapsed lung or lobe. In the other 14 the radiograph was unchanged (table 2). Of the 13 perfusion scans obtained before treatment, four (Nos 9, 13, $14,17)$ showed no apparent perfusion of the affected lung; in three of these cases the pretreatment radiograph showed complete or partial collapse of the ipsilateral lung and the fourth patient (No 13) has a hilar mass. Ten days after treatment only one of these four patients (No 13) showed any evidence of perfusion to the affected side, and in this subject the post-treatment contribution of the diseased side was only $4 \%$ of the total perfusion. Two patients (Nos 18 and 20) showed reductions in perfusion to the affected side in the postoperative scan. Overall, in patients 8-20 there was a small mean improvement in relative perfusion-from $10.2 \%$ to $12.65 \%$ of the total, a change that was not statistically significant.

In patients 8-12 qualitative assessment of the ventilation scans showed defects closely matching those of 
perfusion; in the patient (No 8) showing the greatest improvement in perfusion the changes in ventilation at day 10 were similar (fig 3). Of the eight patients (Nos 13-20) in whom a quantitative comparison was made, five (Nos 13,14, 16, 17, 19) showed virtually no ventilation of the diseased lung before treatment and in only one of these (No 16) was there an obvious increase after treatment.

\section{Discussion}

Our results confirm that carbon dioxide laser resection improves airway function and lessens breathlessness in patients with tumours in the trachea or in a main bronchus. Although some individual patients showed a dramatic improvement in airflow limitation, the overall increase was modest, FEV $_{1}$ increasing by an average of 0.361 and PEF by 391 $\min ^{-1}$. Other studies ${ }^{2}$ have also shown improvement in airway function after laser treatment but differences in selection of patients, the techniques used, and timing of the measurements make comparisons difficult. Eight of the 20 patients felt more breathless on the day after treatment, and at this stage only $\mathrm{FEV}_{1}$ had improved significantly. Failure to improve or deterioration shortly after laser treatment in some patients might result from prolonged anaesthesia or from oedema of the airway.

In only six of the 13 patients with tumours distal to the main carina did the treatment produce an improvement in perfusion of the affected lung. Where the affected lung was not perfused before treatment, no significant function was restored to the diseased side. There are several possible explanations for the small changes in blood flow in our patients. Firstly, seven of the 13 patients had previously had radiotherapy and they may have developed radiation fibrosis or vascular damage in the diseased lung. There was, however, no systematic difference with regard to change in perfusion between those patients who had prior radiotherapy and those who did not. More probably the persisting reduction in function is due to residual tumour, as centrally placed tumours may result in much larger defects in perfusion than would be expected from the radiographic or bronchoscopic appearances. ${ }^{4}$

Although we did not set out to compare the functional effects of laser treatment and radiotherapy, our finding of improved airway function differs from the
Gilmartin, Veale, Cooper, Keavey, Gibson, Morrit! results of Saunders et al, ${ }^{8}$ who showed no improve ment in tests of airway function in nine patients wit tumours in main or lobar bronchi six weeks after. radiotherapy. By contrast, Fazio et $a l^{9}$ reported sub? stantial improvement in ventilation and perfusio $\AA$ scans after radiotherapy for central carcinomas. Th: improvements in perfusion were larger than we found but nearly half of their patients had small cell lung cancer, which is more responsive to radiotherapy than are other histological types. A direct comparison of laser resection and radiotherapy in a previously untreated group of patients with central tumours would therefore be worthwhile.

In conclusion, we have found that laser treatmenif improves airway function and lessens breathlessnesf in patients with tumours in the trachea or either main bronchus. In unilateral disease changes in perfusion of the affected lung were variable and usually small. WE did not, however, find any evidence to suggest that improvements in ventilation after laser treatment would lead to a disadvantageous increase in deade space.

\section{References}

1 Dumon JF, Reboud E, Garbe L, Aucomte F, Meric B Treatment of tracheobronchial lesions by laser photo $\frac{\text { }}{\mathbb{Q}}$ resection. Chest 1982;81:278-84.

2 Hetzel MR, Nixon C, Edmonstone WM, et al. Lase $\overrightarrow{\bar{\sigma}}$ therapy in 100 tracheobronchial tumours. Thorax 1985;40:341-5.

3 Garnett ES, Goddard BA, Fraser HS, MacLeod WM Lung perfusion patterns in carcinoma of bronchus Br Med J 1968;ii:209-10.

4 Secker-Walker RH, Alderson PO, Wilhelm J, Hill RL@ Markham J, Kinzie J. Ventilation-perfusion scanning in carcinoma of the bronchus. Chest 1974;65:660-3.

5 Quanjer PH, ed. Standardised lung function testing. Bulth Eur Physiopathol Respir 1983;suppl 5.

6 Bass $H$. The flow volume loop: normal standards and abnormalities in chronic obstructive pulmonary dis윽 ease. Chest 1973;63:171-6.

7 Gelb F, Epstein JD. Laser treatment of lung cancer을 Chest 1984;86:662-6.

8 Saunders KB, Rudolf M, Banks RA, Riordon JF. Cenज tral airways obstruction in carcinoma of the bronchus treated by radiotherapy: a study of pulmonary func N tion. Br J Radiol 1978;51:286-90.

9 Fazio F, Pratt TA, McKenzie CG, Steiner RE Improvement in regional ventilation and perfusiote after radiotherapy for unresectable carcinoma of theD bronchus. AJR 1979;133:191-200. 